\title{
Interpolation de la formule de Colebrook pour l'application de la méthode de Hardy Cross aux réseaux de distribution d'eau
}

\section{Interpolation of the Colebrook formula for applying the Hardy Cross method to water distribution systems}

\author{
Gianfranco Rossi † \\ Professeur à l'Université de Bologne \\ Vice-Président de l'Association nationale de l'industrie du gaz en Italie
}

On sait que la vérification des réseaux hydrauliques maillés selon la méthode de Hardy Cross pour l'équilibrage des charges, tant dans la procédure par itération maille par maille que dans le développement dérivé, se base essentiellement sur les points suivants :

- on établit un sens de rotation pour les différentes mailles marquées par le numéro d'origine $i$;

- le débit $Q_{i j}$ du côté $j$ appartenant à la maille $i$ est considéré comme étant positif s'il concorde avec ce sens, négatif dans le sens contraire;

- la valeur absolue de la perte de charge $y$ est exprimée par $y=f(|Q|)$, avec le même signe que le débit $Q$; l'expression de telles pertes en valeur et en signe est la suivante :

$$
Y=\varphi(Q)=f(|Q|) \cdot \frac{\psi}{|Q|}
$$

Il est évident que le paramètre typique des fonctions $f(|Q|)$ et $\varphi(Q)$ varie suivant le côté;

- on choisit tout d'abord, pour le réseau, un ensemble de débits $Q_{i j}$ compatibles aux nœuds, mais pas forcément équilibré le long des mailles donc le long de la maille $i$ résultera normalement

$$
\sum_{j=m}^{n} \varphi_{i j}\left(Q_{i j}\right) \neq 0
$$

$m$ et $n$ représentant les valeurs extrêmes des numéros d'ordre $j$ des côtés de la maille $i$ et $\varphi_{i j}$ indiquent la fonction "pertes de charge " en valeur et signe avec les paramètres spécifiques du côté $j$ de la maille $i$;

- on veut déterminer pour chaque maille $i$ un débit de corrections $q_{i}$, circulant le long de la même maille tel que

$$
\sum_{j=m}^{n} \varphi_{i j}\left(Q_{i j}+q_{i}\right)=0 .
$$

En développant l'expression sous le signe somme en série de Taylor (limitée par approximation aux deux premiers termes) et en exécutant une succession de passages, on arrive à l'expression approximative du débit correctif ci-dessous

$$
\begin{gathered}
q_{i}=-\frac{\sum_{j=m}^{n} \varphi_{i j}\left(Q_{i j}\right)}{\sum_{j=m}^{n} f^{\prime}{ }_{i j}\left(\left|Q_{i j}\right|\right)} \\
f^{\prime}{ }_{i j}(|Q|)=\frac{d f_{i j}(|Q|)}{d|Q|}
\end{gathered}
$$

Par exemple, pour

$$
f_{i j}(\mid Q)=R_{i j}|Q|^{\alpha_{i j}}
$$

où $R_{i j}$ est la résistance hydraulique du côté $j$ de la maille $i$, il résulte

$$
\begin{aligned}
& \varphi_{i j}(Q)=R_{i j}|Q|^{\alpha_{i j}} \cdot Q \\
& f^{\prime}{ }_{i j}(|Q|)=\alpha_{i j} R_{i j}|Q|^{\alpha_{i j}-1}
\end{aligned}
$$

donc

$$
q_{i}=-\frac{\sum_{j=m}^{n} R_{i j}\left|Q_{i j}\right|^{a_{i j-1}} \cdot Q_{i j}}{\sum_{j=m}^{n} \alpha_{i j} R_{i j}\left|Q_{i j}\right|^{\alpha_{i j-1}}}
$$

Dans cette étude, on adopte comme fonction représentative de la pente piézométrique dans une conduite parcourue par de l'eau en mouvement uniforme et en régime turbulent, la formule suivante

$$
J=\frac{\lambda V^{2}}{2 g D}
$$

où :

$J=$ pente piézométrique en $\mathrm{m} / \mathrm{m}$

$V=$ vitesse moyenne de leau dans la section en $\mathrm{m} / \mathrm{s}$

$D=$ diamètre intérieur de la conduite en $\mathrm{m}$

$g=$ accélération de la pesanteur $=9,81 \mathrm{~m} / \mathrm{s}^{2}$

$\lambda=$ coefficient de frottement (sans dimension) exprimé par la formule de Colebrook 


$$
\frac{1}{\sqrt{\lambda}}=-2 \log \left(\frac{2,51}{\operatorname{Re} \sqrt{\lambda}}+\frac{\varepsilon}{3,71 D}\right)
$$

$\varepsilon=$ valeur de la rugosité en $\mathrm{m}$

$\mathrm{Re}=$ nombre de Reynolds (sans dimension), exprimé par la formule $\mathrm{Re}=V D / v$ où $v$ est la viscosité cinématique du fluide en $\mathrm{m}^{2} / \mathrm{s}$.

Une fois que l'on a choisi pour l'eau une température de référence égale à $12^{\circ} \mathrm{C}$, et $v=1,236 \cdot 10^{-6} \mathrm{~m}^{2} / \mathrm{s}$, il en résulte

$$
\frac{1}{\sqrt{\lambda}}=-2 \log \left(\frac{3,102 \cdot 10^{-6}}{V D \sqrt{\lambda}}+\frac{\varepsilon}{3,71 D}\right)
$$

La structure d'une telle formule se prête mal à son utilisation pour l'application de la méthode de Hardy Cross aux réseaux de distribution d'eau.

C'est pour cette raison qu'on a tout d'abord calculé et mis en tableau une série de valeurs de $100 \lambda$ (la multiplication par 100 a été faite pour des raisons de commodité) en fonction de $\varepsilon, D$ et $V$ dans les domaines de variation qui intéressent les réseaux de distribution d'eau, c'est-à-dire,

$$
\begin{gathered}
0 \leqslant \varepsilon \leqslant 2,5 \cdot 10^{-3} \mathrm{~m} \\
0,5 \leqslant V \leqslant 2 \mathrm{~m} / \mathrm{sec} . \\
0,1 \leqslant D \leqslant 1 \mathrm{~m}
\end{gathered}
$$

et par conséquent $40453 \leqslant \operatorname{Re} \leqslant 1618123$,

en mettant davantage de données en face des valeurs basses des variables, où la variation de $\lambda$ augmente.

Ces valeurs sont portées dans le tableau $I$, et figurent en première ligne à partir du haut dans les cases correspondantes. Il faut rema: quer à ce propos que dans la colonne pour $10^{3} \varepsilon=0,03$, on a indiqué les valeurs correspondantes de $100 \lambda$ uniquement pour information. Elles ne seront pas utilisées dans les développements qui suivront. Ces valeurs figurent donc entre parenthèses.

Tenant compte de ce que l'on vient de dire, nous avons entrepris l'interpolation des valeurs du tableau $I$ en fonction de $\varepsilon, V$ et $D$ comme fonction du type

$$
100 \lambda=\mu(\varepsilon) \cdot V^{\zeta(c)} \cdot D^{\Re(\varepsilon)}
$$

où $\mu(\varepsilon), \zeta(\varepsilon)$ et $\vartheta(\varepsilon)$ représentent trois fonctions de $\varepsilon$. Elles sont pourtant constantes dans le domaine de chaque colonne du tableau I.

Dans ces conditions, pour chacune de ces colonnes (en excluant comme on l'a déjà dit la colonne correspondant à $10^{3} \varepsilon=0,03$ pour ne pas exagérer le poids des valeurs basses de $\varepsilon$ ), on a effectué l'interpolation des données par la fonction

$$
100 \lambda=\mu \cdot V^{5} \cdot D^{3}
$$

en calculant, sur machine électronique, les valeurs des paramètres $\mu$, $\zeta$ et $\vartheta$ par la méthode des moindres carrés sur la base de la condition

$$
\Sigma_{i}\left(100 \lambda_{i}-\mu V_{i}^{\zeta} D_{i}^{9}\right)^{2}=\min
$$

où $100 \lambda_{i}, V_{i}$ et $D_{i}$ représentent les groupes de données correspondant à la colonne intéressée.

Les valeurs ainsi obtenues pour les paramètres sont reportées au pied de chaque colonne du tableau $I$ et figurent en première ligne dans les cases correspondantes.
On a donc procédé à l'interpolation de $\mu, \zeta$ et $\vartheta$ en fonction de $\varepsilon$, en choisissant après différentes tentatives les fonctions suivantes

$$
\mu=\exp \left[\left(a \varepsilon+b_{0}^{1 / c}\right)^{c}\right]
$$

où $b_{0}=\ln \mu_{0}$

$\mu_{0}=$ valeur de $\mu$ pour $\varepsilon=0$

$$
\zeta=-\left(d \varepsilon+f_{0}^{1 / g}\right)^{g}
$$

où $f_{0}=-\zeta_{0}$

$\zeta_{0}=$ valeur de $\zeta$ pour $\varepsilon=0$

$$
\vartheta=-\left(h \varepsilon+l_{0}^{1 / m}\right)^{m}
$$

où $l_{0}=-\vartheta_{0}$

$\vartheta_{0}=-$ valeur de $\vartheta$ pour $\varepsilon=0$

Les couples de paramètres $(a, c),(d, g),(h, m)$ ont été déterminées sur machine électronique par la méthode des moindres carrés en imposant les conditions

$$
\begin{aligned}
& \Sigma_{i}\left(\mu_{i}-\mu\right)^{2}=\min \\
& \Sigma_{i}\left(\zeta_{i}-\zeta\right)^{2}=\min \\
& \Sigma_{i}\left(\vartheta_{i}-\vartheta\right)^{2}=\min
\end{aligned}
$$

par $\mu_{i}, \zeta_{i} \vartheta_{i}$, on entend les données du tableau $I$ corresondant à $\varepsilon_{i}$.

On a ainsi obtenu les valeurs suivantes

$$
\begin{aligned}
& a=294 \\
& c=0,334 \quad b_{0}^{1 / c}=(\ln 1,202)^{1 / 0.334}=6,292 \cdot 10^{-3} \\
& d=46,6 \cdot 10^{3} \\
& g=-0,990 \quad f_{0}^{1 / g}=(0,195)^{-1 / 0.990}=5,214 \\
& h=0,548 \\
& m=0,167 \quad l_{0}^{1 / m}=(0,195)^{1 / 0.167}=5,607 \cdot 10^{-5}
\end{aligned}
$$

Les fonctions $\mu(\varepsilon), \zeta(\varepsilon), \vartheta(\varepsilon)$ deviennent donc :

$$
\begin{gathered}
\mu(\varepsilon)=\exp \left[\left(294 \varepsilon+6,292 \cdot 10^{-3}\right)^{0.334}\right] \\
\zeta(\varepsilon)=-\left(46,6 \cdot 10^{3} \varepsilon+5,214\right)^{-0.990} \\
\vartheta(\varepsilon)=-\left(0,548 \varepsilon+5,607 \cdot 10^{-5}\right)^{0.167}
\end{gathered}
$$

Leurs valeurs en fonction de $\varepsilon$ sont reportées à titre de comparaison dans les cases correspondantes en $2^{c}$ ligne au pied du tableau $I$.

On a ainsi atteint le but fondamental de cette étude, à savoir l'interpolation avec une fonction du type (8) des valeurs de $\lambda$ données par la formule de Colebrook dans le domaine des valeurs de $\varepsilon$, de $V$ et de $D$ qui intéressent les réseaux de distribution d'eau. Cette fonction s'écrit

$$
100 \lambda=\mu(\varepsilon) \cdot V^{\xi(c)} \cdot D^{\Re(c)}
$$

avec les valeurs de $\mu$, $\zeta$ et $\vartheta$ ci-dessus.

Les valeurs calculées par la fonction d'interpolation (9) sont reportées en $2^{e}$ ligne dans les cases du tableau $I$, où figurent aussi en $3^{\text {e }}$ ligne et avec leur signe, les écarts en pourcentage entre les valeurs d'origine de $100 \lambda$ et les valeurs interpolées.

L'écart quadratique moyen entre les deux séries de valeurs (en excluant la colonne correspondant à $10^{3} \varepsilon=0,03$ ) est de $1,373 \%$, tandis que les écarts extrêmes sont de $+3,03 \%$ et de $-2,94 \%$. 
Tableau 1. - Valeurs de $100 \lambda$, calculées en fonction de $\varepsilon, D, V$, selon la formule de Colebrook (7) (en première ligne dans les cases), selon la fonction d'interpolation (9) (en dernière ligne), et les écarts relatifs en pourcentage (en troisième ligne avec le signe) Paramètre d'interpolation.

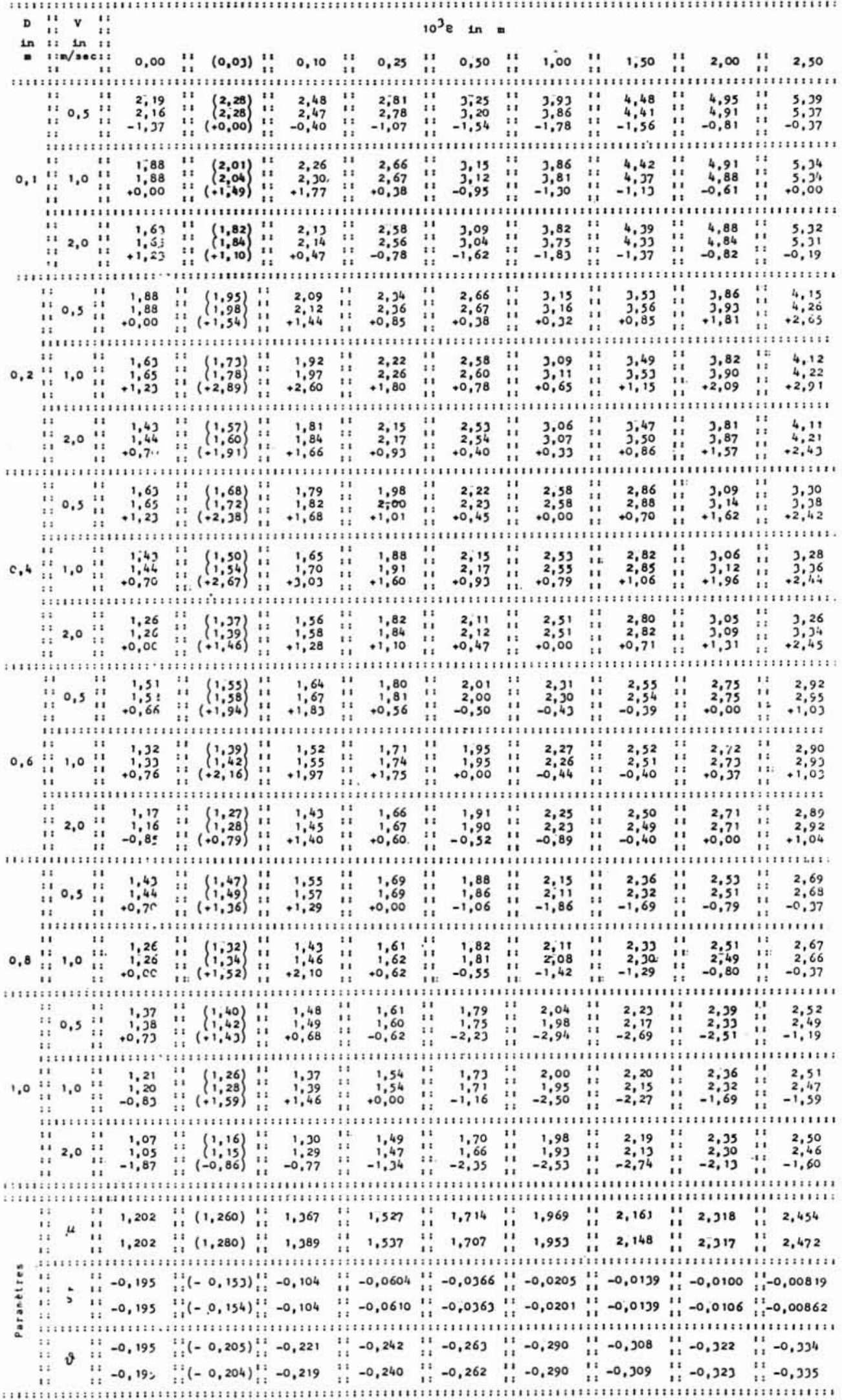


Tableau II. - Exemples de valeurs de $\alpha$, Bet $\gamma$ en fonction de $10^{3} \cdot \varepsilon$, à insérer dans la formule (10).

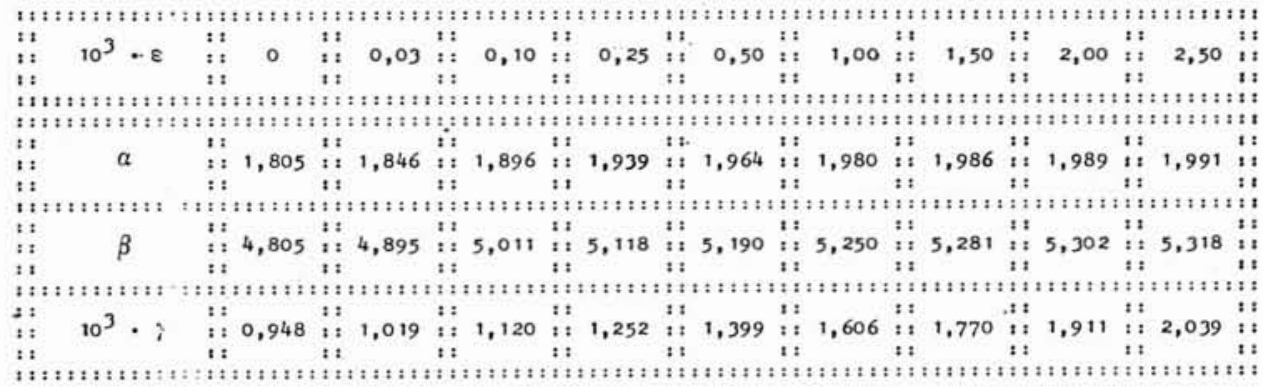

On constate ainsi l'excellent degré d'approximation de la fonction d'interpolation qui, comme on l'a déjà dit, couvre à elle seule tout le domaine des réseaux de distribution d'eau.

Pour une conduite donnée, ayant établi la valeur de $\varepsilon$ en fonction de la nature du matériau et de l'état de conservation de la surface intérieure, les fonctions prennent les valeurs numériques $\mu, \zeta, \vartheta$ et $100 \lambda$ de sorte que la pente piézométrique en fonction de $V$ et $D$ est donnée par la formule (6)

$$
J=\lambda \frac{V^{2}}{2 g D}=e^{\ln \mu-7.582} \cdot \frac{V^{2+\zeta}}{D^{1-8}}
$$

on a en effet

$$
\ln \frac{1}{100 \cdot 2 g}=-7,582
$$

et

$$
V=\frac{4 Q}{\pi D^{2}}
$$

$Q=$ débit en $\mathrm{m}^{3} / \mathrm{s}$

la pente piézométrique en fonction de $Q$ et de $D$, est exprimée par la formule

$$
J=e^{\ln \mu+0.2425-7.098} \cdot \frac{Q^{2+5}}{D^{5-9+25}}
$$

en effet

$$
\ln (4 / \pi)=0,242 .
$$

Il s'agit d'une expression du type

$$
J=\gamma Q^{\alpha} / D^{\beta}
$$

oủ les paramètres $\alpha, \beta$ et $\gamma$ sont des fonctions de $\varepsilon$ et, plus précisément :

$$
\begin{gathered}
\alpha=2-\left(46,6 \cdot 10^{3} \varepsilon+5,214\right)^{-0.990} \\
\beta=5+\left(0,548 \varepsilon+5,607 \cdot 10^{-5}\right)^{0.167} \\
\gamma=e^{\left(294 \varepsilon+6,29210^{-3,0,334}\right.}-\left(23,14 \cdot 10^{3} \varepsilon+2,589\right)^{-0.990} \\
-\left(195,3 \cdot 10^{3} \varepsilon+21,36\right)^{-0.990}-7,098
\end{gathered}
$$

Si l'on considère n'importe quel côté $j$ de n'importe quelle maille $i$ du réseau, si $L_{i j}$ est la longueur de ce côté (en m), la fonction correspondante " pertes de charge " est en valeur absolue exprimée par la relation

$$
f_{i j}(|Q|)=L_{i j} \cdot \gamma_{i j}|Q|^{\alpha_{i j} / D_{i j}^{\beta_{i j}}}
$$

où $L_{i j} \cdot \gamma_{i j} / D_{i j}^{\beta_{i j}}$ représente la résistance hydraulique $R_{i j}$ du côté; on peut donc écrire comme suit

$$
f_{i j}(|Q|)=R_{i j}|Q|^{a_{i j}}
$$

On est donc arrivé à une expression conforme à la formule (2) qui permet le développement des formules (3), (4) et (5).

Il en résulte que la fonction d'interpolation (9), dont on a constaté l'excellent degré d'approximation à la formule de Colebrook dans le domaine des valeurs de $\varepsilon, V$ et $D$ qui intéressent les réseaux de distribution d'eau, est en même temps assez souple pour être utilisée selon la méthode de Hardy Cross selon la formule (10) qui en est la conséquence directe.

Pour finir, on indique en exemple dans le tableau $I I$ les valeurs de $\alpha$, de $\beta$ et de $\gamma$ que l'on doit insérer dans la formule (10) pour quelques valeurs typiques de $\varepsilon$ toujours dans le cas de l'eau à $12^{\circ} \mathrm{C}$.
Le Professeur Rossi est décédé à Bologne, le 13 septembre 1984. à l'issue d'une longue bataille qu'il a livrée contre la maladie. Monsieur Gianfranco Rossi était ingénieur diplômé de l'École Polytechnique de Turin. Au cours des années 1960, il a exercé les fonctions de Directeur Général de la Régie du gaz de Brescia. Il a ensuite, été nommé Vice-Président-Directeur Général de la Régie du gaz de Naples, ville dans laquelle il a travaillé dans le courant des années 1970. Son activité gazière l'a porté à la vice-présidence de l'Association nationale du gaz en Italie.
Par ailleurs, Monsieur Rossi, passionné par l'enseignement, était professeur d'hydraulique à l'Université de Bologne.

Je l'ai connu dans le cadre de travaux effectués à l'Union internationale de l'industrie du gaz au sein de laquelle il a assuré la présidence de la sous-commission chargée des études sur l'association du gaz avec les énergies renouvelables.

Sa forte personnalité, sa profonde culture et sa passion pour la science et la technique resteront à jamais dans nos mémoires.

J.R. Fellous 